\title{
Prolonged Fever in Adult Still's Disease - A Case Report and Review of Literature
}

\begin{abstract}
MSH MAJUMDER ${ }^{a}$, ME JALIL ${ }^{b}$
Summary:

Prolonged high fever is a common clinical problem and sometimes the diagnosis is difficult, management can be a challenge to the clinicians. Adult onset Still's Disease is a rare systemic inflammatory disorder of unknown etiology that is responsible for a significant proportion of cases of fever of unknown origin and can also have serious musculoskeletal sequelae. The disease presents as a diagnostic and therapeutic challenge to the clinicians and

Introduction:

In 1897, George Still described a special form of arthritis in children, what is today called Still's disease. In Still's original description, he attempted to distinguish a form of chronic joint disease in children from rheumatoid arthritis in adults. The distinct features included pyrexia, splenomegaly and enlargement of lymph nodes. Still's did not describe the presence of rash, an important feature in the diagnosis of Still's disease today. The similar illnesses were found in adults and reported by many clinicians, some of which as undiagnosed fever or pyrexia of unknown origin. The first use of the term "adult Still's disease" was by Eric Bywaters, the eminent English Rheumatologist in 1966. ${ }^{1}$

clinical guidelines are lacking. The emergence of classification criteria, discovery of better serological markers and the application of new biological agents may all provide the clinicians with significant tools for the diagnosis and management of this complex disorder.

Key words: Adult Still's Disease (ASD), Adult-onset Still's Disease (AOSD), arthritis, ferritin

(J Banagladesh Coll Phys Surg 2014; 32: 224-228)

leucocytosis. It is frequently underdiagnosed and remains one of the main reasons for hospital admissions due to pyrexia of unknown origin. ${ }^{2}$ The disease commonly affects young individuals between 16 and 35 years of age and male and female are affected equally. ${ }^{3}$ The clinical picture is variable with mild to life threating courses. The disease is self-limiting, intermittently active or chronic. ${ }^{4}$ Diagnosis is clinical and may be lengthy because it requires exclusion of infectious diseases, neoplasms including lymphoma and leukaemias, and autoimmune diseases. High serum ferritin levels associated with a low fraction of its glycosylated component are useful diagnostic and disease activity markers. ${ }^{5}$
\end{abstract}

Adult Still's disease (ASD) or adult-onset Still's disease (AOSD) is a rare, immune-mediated, multisystem inflammatory disorder of unknown etiology characterized by daily high spiking fever, evanescent rash and arthritis, frequently accompanied by sore throat, lymphadenopathy, splenomegaly and neutrophillic

a. Prof. Md. Shafayet Hasan Majumder, Professor, Department of Medicine, Medical College for Women \& Hospital, Uttara, Dhaka

b. Dr. Md. Ehsan Jalil, Senior Medical Officer, Department of Medicine, Medical College for Women \& Hospital, Uttara, Dhaka.

Address of Correspondence: Prof. Md. Shafayet Hasan Majumder, Professor, Department of Medicine, Medical College for Women \& Hospital, Uttara, Dhaka

Received: 18 September, 2013

Accepted: 20 May, 2014

\section{Case Report:}

A 22 - year old married female, short stature, housewife, low socioeconomic status, mother of a one year child, from a rural area of Bangladesh, was admitted into our female medical ward with history of high intermittent fever with chills and rigor, polyarthralgia, sore throat, bodyache and weight loss for 3 months. The fever was high spiking, 103 degree $\mathrm{F}$ or above, rose rapidly in the evening and then subsided to or near normal by several hours or the next morning (Fig.-1a). In some days, two fever spikes were noticed. Her sore throat was described as a severe, constant burning pain in the area around pharynx, more prominent during febrile period. Physical examination showed an emaciated (weight- $29 \mathrm{~kg}$ ), toxic girl with moderate anemia, no skin rashes, swollen and tender both wrists, left ankle joint, cervical 


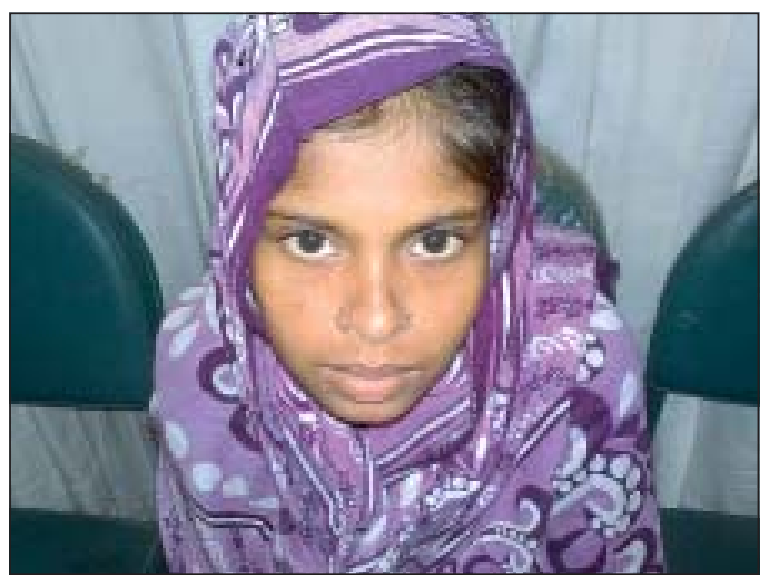

Fig.-1: Photograph of the Patient

lymphadenopathy , mildly enlarged non-tender liver and splenomegaly.Cardiovascular and respiratory system examination revealed normal findings. Deep cervical lymph nodes in the left anterior triangle of the neck were palpable, 3 in number, size $1-2 \mathrm{~cm}$ in diameter, soft in consistency, non tender, freely mobile and not fixed to underlying structures or overlying skin. During her long febrile period, she was treated with phenoxymethylpenicillin, levofloxacin, doxycycline, cefixime, antimalarial drugs before hospital admission without any benefits. Her laboratory investigations were as follows : Complete blood count \& peripheral blood film - Hb $9.3 \mathrm{gm} / \mathrm{dl}$, ESR $106 \mathrm{~mm} / 1^{\text {st }}$ hour , RBC3.81million/cumm, MCV-74fl, MCH- 24pg, WBC30,000/cumm (Neutrophil - 87\% , L -11\%, M - 2\% E - 0\% ), Platelet - 487,000/cumm , Malaria parasite not found ; Widal Test - T O - 1:80, T H - 1:80, A O 1:40, A H-1:40, B O- 1:40, В H- 1:40, and after 2 weeks, no rising titre ; blood culture twice yielded no growth of any bacteria, ASO Titre - $107 \mathrm{IU} / \mathrm{ml}$, S. Creatinine - 0.85 mg/dl , Random blood sugar - 6.2 mmol/L;
Urinalysis - normal ; Urine Culture repeatedly - no growth. X-ray chest, ECG and transthoracic echocardiography showed normal findings. Abdominal ultrasound revealed normal findings except mild hepatosplenomegaly. Following hospital admission, she was treated empirically with quinine and then ceftriaxon without any improvement. As the patient was not recovering, she was further investigated, some investigations were repeated and the results were as follows : Complete blood count - $\mathrm{Hb} 8.6 \mathrm{gm} / \mathrm{dl}$, ESR $108 \mathrm{~mm} / 1^{\text {st }}$ hour , WBC -26,000/cumm (N -82\%, L16\%, M - 01\%, E-01\%) ; R A Test - negative, ANA - negative, Anti CCP - negative, CRP - 76 mg/L , VDRL - nonreactive, Tuberculin Test - 05 mm, S. Ferritin - 2400 ng/ml(highly elevated), S Bilirubin$0.4 \mathrm{mg} / \mathrm{dl}$, S. ALT- 26U/L, Urinalysis - normal , S. Fibrinogen- 394 mg/dl , X-ray chest and paranasal sinuses - normal. Serum protein and haemoglobin electrophoresis, bone marrow aspiration was under plan and waiting. Infectious and neoplastic causes of her illnesses were tried to be ruled out by a thorough clinical and laboratory reports evaluation.

Literatures were reviewed and a diagnosis of Adult onset Still's Disease(AOSD) was made considering Yamaguchi criteria. ${ }^{6}$ Tablet prednisolone(1mg/kg/day) and methotrexate(7.5mg weekly) orally were started. Her condition was dramatically improved. She became afebrile (Fig.-1b), appetite and general condition improved.

Subsequent investigations after 6 weeks showed the following : CBC Hb-10.2g/dl, WBC-14000/cumm(N$70 \%, \mathrm{~L}-25 \%, \mathrm{M}-4 \%, \mathrm{E}-1 \%), \mathrm{ESR}-15 \mathrm{~mm}$ in $1^{\text {st }}$ hour, CRP- $6 \mathrm{mg} / \mathrm{L}$.

Follow up visit after 6 month : Healthy smiling girl with mildly tender both wrists with slightly restricted

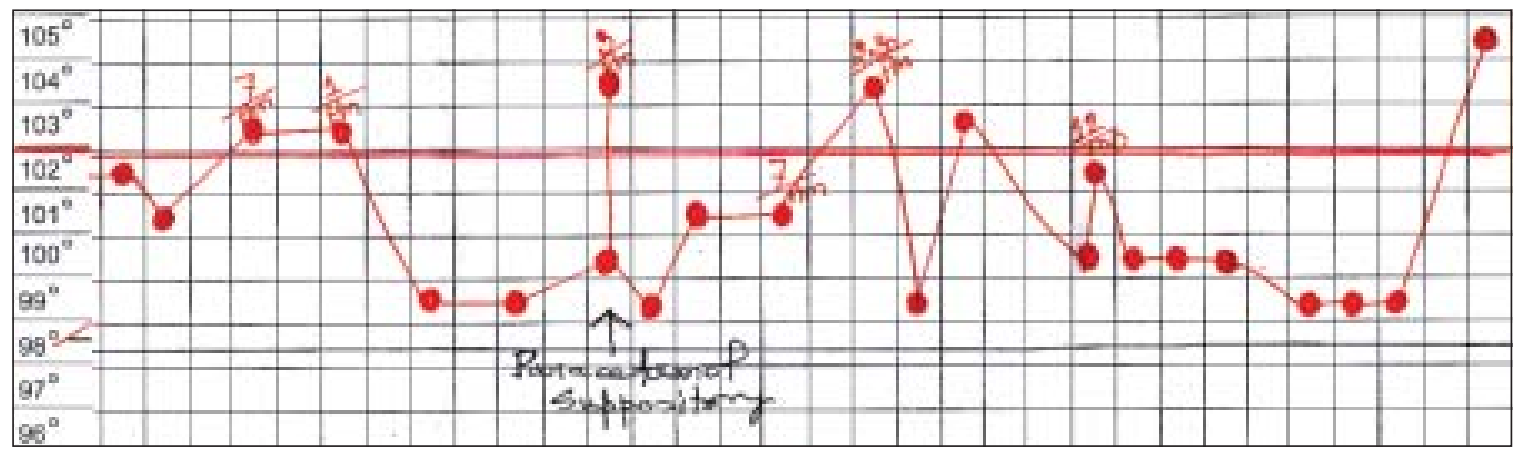

Fig.-2(a): Daily high spiking fever recorded (some part) in this patient. 


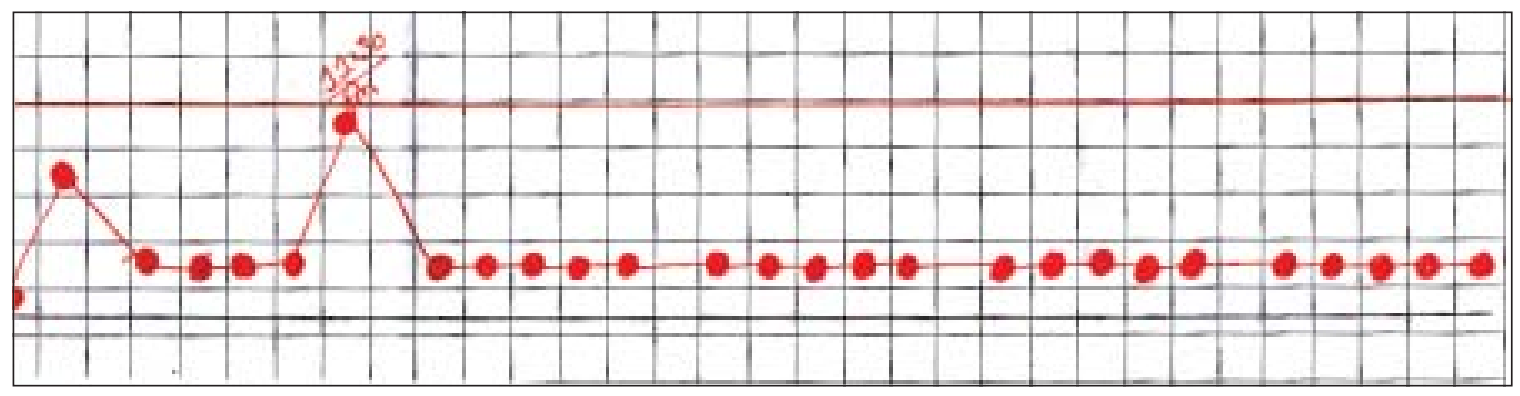

Fig.-2(b): The fever subsided after the start of corticosteroid treatment.

movements; regularly taking methotrxtate 7.5mg weekly orally and laboratory investigations as $\mathrm{Hb} 11.1 \mathrm{~g} / \mathrm{dl}$, ESR $20 \mathrm{~mm}$ in $1^{\text {st }}$ hour, CRP $6 \mathrm{mg} / \mathrm{L}$, S. Ferritin - 11.5ng/ml.

Follow up visit after 2 years : Reasonably good health but mild tenderness involving both wrists on passive movement, no fever and no other symptoms, CBC- Hb12.7g/dl, WBC- 13660/cumm ( N-70\%, L- 24\%, M06\%), ESR- $10 \mathrm{~mm}$ in $1^{\text {st }}$ hour, CRP - 3mg/L, ANANegative, S. Ferritin- 18.2ng/L.

\section{Discussion:}

Data on diagnosis and treatment of Adult Still's Disease are limited in medical literature and consists of mainly case reports, small series and some retrospective studies; current knowledge is largely descriptive. Diagnosis is clinical and requires exclusion of infectious, neoplastic and connective tissue diseases. Laboratory tests are nonspecific and show increased immunological response. , $^{3,8}$ There is a speculation that the disease has features of nonnecrotising immune complex vasculitis. ${ }^{1}$ The disease occurs throughout the world, typically affects $16-35$ years old and presents with fever, arthralgia, and sore throat, and skin rash. The fever is typically high and spiking, usually quotidian and occasionally double quotidian. The spike occurs in the late afternoon or evening. The duration of fever is typically brief, and the temperature falls rapidly. ${ }^{3,} 9$

The majority of the patients with AOSD have arthralgia and arthritis, with incidences ranging from $64 \%$ to $100 \%$. Joints affected most frequently are the knees, wrists, ankles, although involvement of elbow, shoulder and small joints of hands are also described. Usually, several joints are involved at the same time. Often, patients have morning stiffness of joints that lasts for several hours. 3,10
The rash is perhaps the most helpful feature in the diagnosis of Still's disease, especially when present in association with high fever and arthralgia. It is predominantly a truncal rash but can spread to the arms and legs including the palms and soles, but usually does not involve the face. The typical eruption is a salmonpink macular or maculopapular rash beginning as small macules that may coalesce; the rash usually is not pruritic, evanescent and may be missed. Patients may not notice it. ${ }^{1,11-13}$ We did not notice any skin rash in this patient, probably because of black complexion.

\section{Diagnosis of Adult Still's Disease}

There is no single diagnostic test for Adult Still's disease and the diagnosis is clinical and not based on serology. Response to empirical corticosteroid therapy generally helps in diagnosis. ${ }^{11}$ Laboratory investigations reveals high WBC count with neutrophilic leukocytosis, raised ESR, high CRP, very high ferritin, high level of AST, ALT and Fibrinogen reflecting systemic inflammation. Rheumatoid factor (RF) and antinuclear antibody (ANA) are classically negative. ${ }^{3,14}$

Recently, serum ferritin and glycosylated ferritin have received a lot of attention as diagnostic and disease activity marker. The glycosylated fraction of ferritin is a more specific marker of AOSD than ferritin itself. In this patient, glycosylated ferritin was not estimated due to unavailability. In healthy subjects, $50-80 \%$ of ferritin is glycosylated; in inflammatory diseases, $20-50 \%$ of ferritin is glycosylated and in AOSD, less than $20 \%$ is glycosylated. ${ }^{3,15}$ Cytokines, such as interleukin(IL)-1, IL-6, interferon(IFN)-gamma, and tumor necrosis factor-alpha, are elevated in patients with AOSD. ${ }^{16}$

At least 7 sets of classification criteria have been devised; of which Yamaguchi, Cush and Fautrel criteria are important. However, the Yamaguchi criteria have 
the highest sensitivity. Diagnosis requires at least 5 criteria, with at least 2 of these being major diagnostic criteria. $^{3}$

\section{Major criteria}

- Fever of at least 39 degree centigrade for at least one week

- Arthralgia or arthritis for at least two weeks

- Nonpruritic salmon colored rash (usually over trunk or extremities while febrile)

- Leukocytosis (10000/microL or greater), with granulocyte predominance

\section{Minor criteria}

- Sore throat

- Lymphadenopathy

- Hepatomegaly or splenomegaly

- Abnormal liver function tests

- Negative tests for antinuclear antibody and rheumatoid factor

\section{Exclusion criteria}

- Infection

- Malignancies

- Other rheumatic diseases

The reported case had almost all the features of Yamaguchi criteria. Skin rash was not obvious, probably because of black complexion. So, a diagnosis of Adult onset Still's Disease was made.

Treatment of AOSD includes NSAID, corticosteroids, immunosuppressive drugs and biological agents. Immunosuppressants including methotrexate, gold, azathioprine, cyclosporine, leflunomide, tcrolimus, and cyclophosphamide; along with intravenous immunoglobin are tried. Biological agents or anticytokines (e.g. anti-TNF-alpha, anti-IL-1, anti-IL6) have been successfully used in refractory cases. ${ }^{2,}, 16-$ ${ }^{18}$ We treated the patient with NSAID, corticosteroids and methotrexate with satisfactory response.

The clinical course of AOSD can be divided into three main patterns with different prognosis : self-limited or monophasic, intermittent or polycyclic systemic and chronic articular pattern. The reported case seems to be chronic articular pattern. AOSD, though not common, can be from milder form of disease to a life threatening condition. ${ }^{19-21}$

Macrophage-activation syndrome (MAS) is a severe, potentially life threatening complication, associated with Adult-onset Still's disease. The hallmark clinical and laboratory features include high fever, hepatosplenomegaly, lymphadenopathy, pancytopenia, liver dysfunction, disseminated intravascular coagulation, hypofibrinogenamia, hyperferritinaemia and hypertriglyceridaemia. The condition is triggered by a viral infection or a medication. Despite marked systemic inflammation, ESR is depressed because of low fibrinogen level. ${ }^{22,23,24}$ Occasionally, AOSD may be complicated by thrombotic thorombocytopenic purpura and haemolytic uraemic syndrome. ${ }^{25}$

\section{Conclusion:}

Adult onset Still's disease (AOSD) is a rare systemic inflammatory disorder of unknown etiology and pathogenesis, usually presenting with high spiking fever accompanied by systemic manifestations. It is hypothesized that it may be a reactive syndrome where various infectious agents may act as disease triggers in a genetically predisposed host. The suggested etiologies, clinical manifestations and prognoses are diverse. There is no single diagnostic test for AOSD; rather, the diagnosis is based on a set of criteria, the most important of which are indeed clinical. Treatment aims at both minimizing inflammation and halting disease progression. Non-steroidal anti-inflammatory drugs (NSAID), glucocorticoids and disease modifying antirheumatic drugs (DMARD) are used. Novel therapeutic approaches, such as anti-tumor necrosis factor blockade and stem cell transplantation, monoclonal antibodies, may be effective.

\section{References:}

1. Larson E B. Adult Still's Disease - Recognition of a clinical syndrome and recent experience. West J Med 1985 May; 142(5) : $665-671$

2. Kontzias A , Efthimiou P. Adult-onset Still's disease: pathogenesis, clinical manifestations and therapeutic advances. Drugs 2008 ; 68(3): 319-37

3. Efthimiou P, Paik PK, Bielory L. Diagnosis and management of adult-onset Still's disease. Ann Rheum Dis 2006 May; 65(5): 564-572

4. Dechant C, Kruger K. Adult- onset Still's disease. Dtsch Med Wochenschr 2011 Aug; 136(33): 1669-73 
5. Bagnari V, Colina M, Ciancio G, Govoni M, Trotta F. Adult onset Still's disease. Rheumatol Int 2010 May;30(7): 855-62

6. Yamaguchi M, Ohta A, Tsunematsu T, Kasukawa R, Mizushima Y, Kashiwagi H, Kashiwazaki S, Tanimoto K, Matsumoto Y, Ota T, et al. Preliminary criteria for classification of adult Still's disease. J Rheumatol. 1992 Mar;19(3):424-30

7. Cabanelas N, Ferreira P, Esteves MC, Roxo F. New insights in adult Still's disease's knowledge. Acta Med Port 2011 JanFeb; 24(1): 183-92

8. Vanderschueren S, Hermans F, De M P, Knockaert D. Adultonset Still's disease: still a diagnosis of exclusion. A nested case-control study in patients with fever of unknown origin. Clin Exp Rheumatol 2012 Mar 16

9. Mehta B, Efthimiou P. Ferritin in Adult-Onset Still's Disease: Just a Useful Innocent Bystander? International Journal of Inflammation 2012

10. Riera E, Olive A, Narvaez J, Holgado S, Santo P, Mateo L, Bianchi MM, Nolla JM. Adult onset Still's disease: review of 41 cases. Clin Exp Rheumatol 2011 Mar-Apr; 29(2): 331-6

11. Kadar J, Petrovicz E. Adult-onset Still's disease. Best Pract Res Clin Rheumatol 2004 Oct; 18(5):663-76

12. Ribi C. Adult onset Still's disease. Rev Med Suisse 2008 Apr 23; 4(154): 1039-44

13. Owlia M B, Mehrpoor G. Adult-onset Still's disease : a review. Indian J Med Sci 2009 May; 63(5): 207-21

14. Ohta A, Yamaguchi M, Kaneokaq H, Nagayoshi T, Hiida M. Adult Still's disease: review of 228 cases from the literature. J Rheumatol 1987 Dec; 14(6) 1139-46

15. Cush J J. Adult Onset Still's Disease: A circadian cytokines syndrome. International Still's Disease Foundation, Inc.
16. Efthimiou P, Georgy S. Pathogenesis and management of adult onset Still's disease. Semin Arthritis Rheum 2006 Dec; 36(3) : 144-52

17. Ahmadi SK, Lamprecht P, Jankowiak C, Gross WL. Successful treatment of refractory adult onset Still's disease with rituximab. Ann Rheum Dis 2006 August; 65(8):1117-1118

18. Cavagna I, Caporali R, Epis O, et al. Infliximab in the treatment of adult Still's disease refractory to conventional therapy. Clin Exp Rheumatol 2001; 19: 319-320

19. Lichauco JJ, Sinha J, Barland P. Adult Onset Still's Disease. Dec 2001. International Still's Disease Foundation

20. Mihaescu R, Serban C, Mozos I, Sirbu E. Adult-onset Still's Disease presenting as persistent fever of unknown origin: case report and review of literature. International Journal of collaborative research on internal medicine \& public health 2013; Vol. 5 No.1: 89-94

21. Cush JJ, Medsger TA Jr, Christy WC, Herbert DC, Cooperstein LA. Adult-onset Still's disease. Clinical course and outcome. Arthritis Rheum. 1987 Feb;30(2):186-94.

22. Grom AA, Mellins ED. Macrophage activation syndrome : advances towards understanding pathogenesis. Curr Opin Rheumatol Sept 2010; 22(5): 561-6.

23. Kato T, Kobayashi T, Nishino H, Hidaka Y. Double-filtration plasmapheresis for resolution of corticosteroid resistant adultonset Still's disease. Clin Rheumatol 2006; 25 : 579-582.

24. Cristina R, Gisele ZG, Esther GMH, David PD, Yehuda S. The hyperferritinaemic syndrome : macrophage activation syndrome, Still's disease, septic shock and catastrophic antiphospholipid syndrome. BMC Medicine 2013, 11 :185.

25. Gopal M, Cohn CD, McEntire MR, Alpecin JB. Thrombotic thrombocytopenic purpura and adult onset Still's disease. Am J Med Sci 2009 May; 337(5) : 373 - 376. 